\title{
The Optimal Control Policy of Dynamic Pricing with Reference Effect
}

\author{
Hui Yang ${ }^{*}$ and Hui Jin \\ School of Economics and Management, Nanjing University of Science and Technology, Nanjing, China \\ ${ }^{*}$ Corresponding author
}

\begin{abstract}
This paper focuses on dynamic pricing decision with a static reference price in revenue management situations. Firstly, it constructs the optimal control model with continuous time and continuous prices. Secondly, it defines the concept of multiplicative reference effect, and describes the characteristics of the reference effect and the demand function with multiplicative reference effect. By solving the Hamilton-Jacobi equation, it presents the optimal price path. Finally, it compares the pricing strategies with the GVR model without reference effect under different circumstances through simulation experiments. The results show that the stronger the reference effect is, the more it influences the optimal price and total revenue. The optimal price and revenue increase with the reference price. The markup policy is effective in high demand situations and the markdown policy is effective in low demand situations. In the situations with low demand and strong reference effect, one should adopt demand-oriented strategies. In other situations, one should adopt price-oriented strategies.
\end{abstract}

Keywords-optimal control; pricing policy; reference effect; simulation

\section{INTRODUCTION}

Researches on dynamic pricing policies in revenue management situations have been pursued over two decades [1]. In the pioneering work, Gallego and Ryzin (1994) formulated a continuous time model (GVR model) to obtain the optimal price function depending on the length of horizon and a function of stock level [2]. On the basis of this model, they also have studied the dynamic pricing model of multiproducts [3]. Gallego and $\mathrm{Hu}$ (2014) focuses on single-product problems with competition [4]. Mersereau and Zhang (2012), Levin, et al. (2010) consider strategic customers [6][7]. Chen and Chen (2014) presents a comprehensive review of the dynamic pricing problems [5]. This paper contributes to expand GVR model to a pricing model with reference effect. In our model, consumers perceive price through gains (discounts) or losses (surcharges) relative to a reference price. Popescu and Wu (2007) presents dynamic pricing models with reference effect in the condition of discrete time and unconstrained capacity [8]. However, we explore the continuous-time problem with limited capacity, taking static reference price into consideration. We can find many examples of dynamic pricing decision with static reference price in business practice. For example, the rapid development of high-speed rail in China has brought more choices for travelers. As a result, when the travelers plan to buy airline tickets, they will refer to the high-speed rail fares. And the managers have to adjust the original airline dynamic pricing policies. The remainder of this paper is organized as follows.
Section 2 describes the optimal control model. Section 3 defines the multiplicative reference effect and analyzes demand function. Section 4 presents the optimal solution with exponential demand function. Section 5 presents simulation results. Finally, section 6 offers concluding remarks.

\section{OPTIMAL CONTROL MODEL}

Assume that the firm has a stock $N$ (a nonnegative integer) of items at the beginning of the selling horizon $t=0$ and it has a finite time $T(T>0)$ to sell these products. The salvage value of any unsold items at time $T$ is zero. At the moment of $t$, the price of the product is $p(t)$ is and the inventory is $n(t), t \in[0, T]$. The realized demand is stochastic and modeled as Poisson process with intensity $\lambda(\cdot)$, which is related to the time, the price and the reference price. The demand function will be further described in Section 3. Let $M(t)$ denote the number of items sold up to time $t$, then $d M(t)$ denote the number of selling items in time period $[t, t+\Delta t]$. We denote $d M(t)$ by

$$
d M(t)=\lambda(\cdot) d t, d M(t)=-d n(t)
$$

We define that the price function $p(t)$ is the system control vector and the inventory function $n(t)$ is the system state vector. We denote the system state vector by

$$
\frac{d n(t)}{d t}=\lambda(\cdot), n(0)=N
$$

And we denote the target set constraint which satisfy

$$
\int_{0}^{T} d M(t) \leq N, p(t) \in[0,+\infty)
$$

Given any initial stock $n>0$ and a sales horizon $t>0$ the performance index function can be written as

$$
J(n(t), t)=E\left\lceil\int_{t}^{T} p(\tau) \lambda(\cdot) d \tau\right\rceil
$$

$J(n(t), t)$ denotes the expected revenue in time period $[0, \mathrm{~T}]$ with boundary condition $J(N, 0)=0$. 
Consequently, the optimal pricing path can be obtained by solving the optimal performance index function $J^{*}(n(t), t)$ to find the optimal control strategy $p^{*}(t)$. The HamiltonJacobi equation is

$$
\frac{\partial J^{*}(n(t), t)}{\partial t}=-\max \left\{(t) \lambda(\cdot)-\lambda(\cdot) \Delta J^{*}(n(t), t)\right\}
$$

where $\Delta J^{*}(n(t), t)=J^{*}(n(t), t)-J^{*}(n(t)-1, t)$ denotes the marginal revenue.

\section{DEMAND FUNCTION}

\section{A. Multiplicative Reference Effects}

In this section we describe how consumers make purchase decisions based on price and reference price. Popescu and $\mathrm{Wu}$ (2007) present a type of additive reference effect $R\left(x, p_{\tau}\right)=D\left(p, p_{\tau}\right)-D(p, p)$, which describes customer behaviors via the magnitude of the perceived "gain" or "loss," $x=p_{\tau}-p$ affect quantity demanded relative to a reference point. Differently, we define the multiplicative reference effect.

$$
R\left(x, p_{\tau}\right)=\frac{D\left(p, p_{\tau}\right)}{D(p, p)}
$$

where $D(p, p) \neq 0$.

Alternatively, $R\left(x, p_{\tau}\right)$ measures the multiplicative impact on demand, that is, a consumer's perceived discount/premium $x=p / p_{\tau}$ has a relative influence on demand, comparing with the reference price $p_{\tau}$.This reference effect function has the following properties.

Property1. $R\left(x, p_{\tau}\right) \geq 1$ for $x<1, \quad R\left(x, p_{\tau}\right) \leq 1$ for $x>1$, and $R\left(1, p_{\tau}\right)=1$.

Property2. $R\left(x, p_{\tau}\right)$ is increasing in $p_{\tau}$.

Property3. $R\left(x, p_{\tau}\right)$ is decreasing in $x$.

Compared with the additive effect, the multiplicative demand function has two advantages: Firstly, it reflects the relative amounts of change in demand intuitively. One can easily observe the demand amplification or contraction. Secondly, it can be integrated easily with the nonlinear demand function as a multiplicative factor. We can find many function forms satisfy the three properties, such as the exponential function as written in (3), which is incorporated in our demand function

$$
R\left(x, p_{\tau}\right)=e^{\theta(1-x)}
$$

where $\theta>0$.

\section{B. Demand Function}

We consider exponential demand function $\lambda(p(t), t)=e^{\alpha-\beta p(t)}$ where $\alpha>0, \beta>0$ are arbitrary parameters. Considering the reference effect, the demand function is

$$
\lambda\left(p(t), p_{\tau}, t\right)=\lambda(p(t), t) \cdot R\left(X, p_{\tau}\right)=e^{\alpha+\theta-\left(\beta+\theta / p_{\tau}\right) p(t)}
$$

It can be written as:

$$
\lambda\left(p(t), p_{\tau}, t\right)=e^{a-b\left(p_{\tau}\right) p(t)}
$$

The demand function has the following properties.

Property 1. $\lambda(p(t), t)$ is decreasing in $p(t)$ and increasing in $p_{\tau}$.

Property 2. $\lambda\left(p(t), p_{\tau}, t\right)<\lambda(p(t), t)$ for $p_{\tau}<p(t)$ and $\lambda\left(p(t), p_{\tau}, t\right)>\lambda(p(t), t) \quad$ for $\quad p_{\tau}>p(t) \quad$.Furthermore $\lambda\left(p(t), p_{\tau}, t\right)=\lambda(p(t), t)$ for $p_{\tau}=p(t)$.

\section{SOLUTION}

The Hamilton-Jacobi function is

$$
H=p(t) \lambda(\cdot)-\lambda(\cdot) \Delta J^{*}(n(t), t)
$$

Its optimal conditions are

$$
\frac{\partial H}{\partial p(t)}=0, \frac{\partial^{2} H}{\partial^{2} p(t)}<0
$$

Using (4), we obtain

$$
p^{*}(t)=\frac{1}{b\left(p_{\tau}\right)}+J^{*}(n(t), t)-J^{*}(n(t)-1, t)
$$

Combining with (1), we gain the maximum revenue in the time interval which satisfies

$$
\frac{\partial e^{b\left(p_{\tau}\right) \cdot J^{*}(n(t), t)}}{\partial t}=-e^{a-1} \cdot e^{b\left(p_{\tau}\right) \cdot J^{*}(n(t)-1, t)}
$$

Therefore, we obtain the maximal revenue:

$$
J^{*}(n(t), t)=\frac{1}{b\left(p_{\tau}\right)} \ln \sum_{i=0}^{n(t)}\left(e^{a-1}(T-t)\right)^{i} \frac{1}{i !}
$$

The optimal price $p^{*}(t)$ is given by

$$
p^{*}(t)=\frac{1}{b\left(p_{\tau}\right)}\left(1+\ln \sum_{i=0}^{n(t)}\left(e^{a-1}(T-t)\right)^{i} \frac{1}{i !}-\ln \sum_{i=0}^{n(t)-1}\left(e^{a-1}(T-t)\right)^{i} \frac{1}{i !}\right.
$$


Using (4) we obtain an optimal intensity $\lambda^{*}(n(t), t)$ in the path of this optimal price

$$
\lambda^{*}(n(t), t)=\frac{e^{a-1} \cdot\left(\sum_{i=0}^{n(t)-1}\left(e^{a-1}(T-t)\right)^{i} \frac{1}{i !}\right)}{\left(\sum_{i=0}^{n(t)}\left(e^{a-1}(T-t)\right)^{i} \frac{1}{i !}\right)}
$$

\section{NUMERICAL EXAMPLES}

In this section, we present a set of numerical examples to analyze the impact of reference effect on the total revenue and the price paths. We take the GVR model (without reference effect) as the benchmarking model. The initial inventory is $N=20$ and the market types are different in two dimensions: high vs. low demand and strong vs. weak reference effect.

\section{A. High Demand}

In this case, the parameters of the demand function are $\alpha=3, \beta=0.2$. The firm sold all the items in the sales period. Furthermore, we use two sets of parameters to represent the degree of reference effect. We determine the values of the reference prices referring to the GVR model results without considering reference effects. The price obtained from the GVR model is called the benchmark price.

The steps of simulation are as follows: Firstly, using (8) to calculate the optimal initial arrival rate $\lambda^{*}(N, 0)$, which generates the sold-out timing of the first product $t_{1}$. Using the same method, we can calculate the sold-out timing $t_{i}$ of each product $i \in[1,20]$. Secondly, For each successive period $\left[t_{i}, t_{i}+1\right]$, calculate the optimal price $p^{*}(N-i, t)$, using (7).

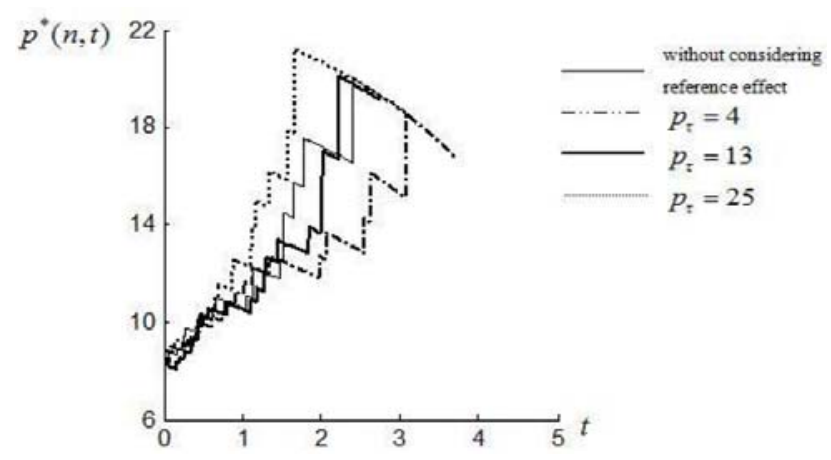

FIGURE I. HIGH DEMAND AND WEAK REFERENCE EFFECT

Consider the case of the weak reference effect $(\theta=0.01)$. $p_{\tau}=4,13,25$ and the initial capacity $n$ ranging from 1 to 20 . The optimal price path is also shown in Figure 1 . We can observe the jumps in the price curve denoting that the products are sold out at the time. Once an item has been sold out, the price decreases gradually until the next item is sold. Figure1. shows that when the reference price is low, the optimal price is lower than the benchmark price and the optimal revenue under the reference effect is reduced by $0.07 \%$, the reason is that the customer makes a purchase behavior without considering the reference price. The optimal price is higher than the benchmark price and the optimal revenue increases by $1.00 \%$ when the reference price is high which is due to the customer's limited rationality. Moreover, when the reference price is in the benchmark interval, the optimal price and revenue remain unchanged.

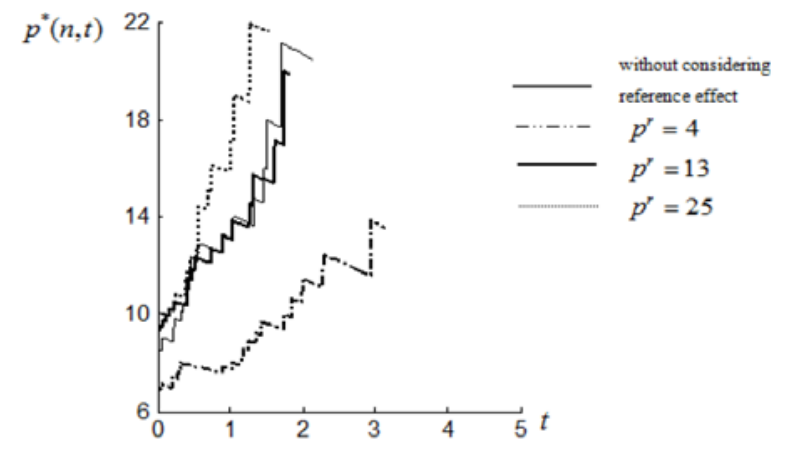

FIGURE II. HIGH DEMAND AND STRONG REFERENCE EFFECT

We next consider the case of strong reference effects $(\theta=0.4)$, Figure 2. shows the price paths. By comparison, we find in this case, the rule of the optimal pricing strategy is similar to the case of weak reference effect. The stronger the reference effect is, the more it influences the optimal prices and the total revenue.

\section{B. Low Demand}

In the low demand situations, the parameters of demand function are set as $\alpha=2, \beta=0.2$. The items are not sold out at the end of selling horizon. Similarly, we use two sets of parameters to represent the weak and strong reference effect. We set $p_{\tau}=3,5,7$ as the reference prices and the benchmark interval is [5.000, 5.004].

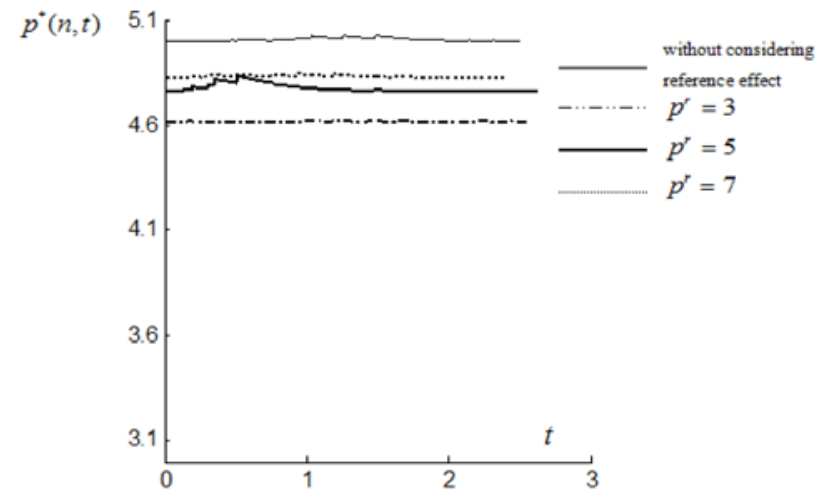

FIGURE III. LOW DEMAND AND WEAK REFERENCE EFFECT

Consider weak reference effect firstly $(\theta=0.05)$, the simulation in this case is shown in Figure 3. The optimal price path has the same characteristics as the case of high demand. However, unlike high demand, when it comes to the end of the consumption period, there is a fall in demand. Therefore, the enterprise improves the revenue by lowering the price to boost demand.

These calculation results is according to what we observe in practice. For example, in the fashion industry of exclusive 
products, the initial price of tickets is usually extremely high. If it is not popular, the price decreases significantly after a couple of weeks. Similarly to the case of high demand, the optimal price here is lower than the benchmark price and the optimal revenue decreases when the reference price is low; the optimal price is higher than the benchmark price and the optimal revenue increases when reference price is high. Moreover, when the reference price is in the benchmark interval, optimal price and revenue remain unchanged.

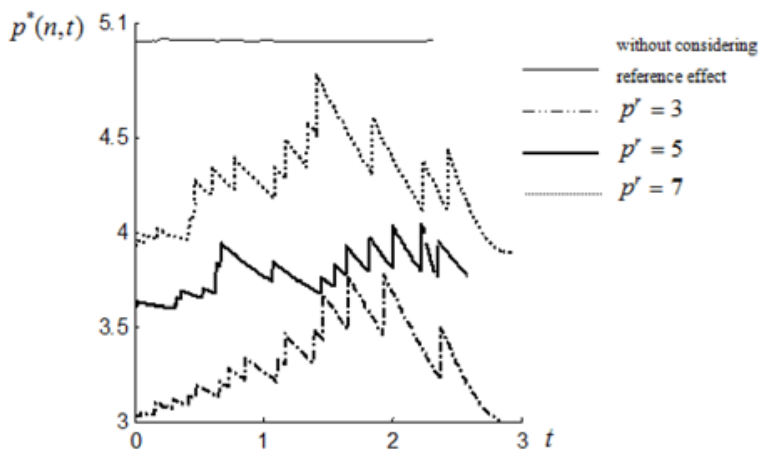

FIGURE IV. LOW DEMAND AND STRONG REFERENCE EFFECT

Considering strong reference effect $(\theta=0.4)$, In Figure4, we note that the optimal price is always lower than the benchmark price no matter how much the reference price or the benchmark price is. This is different from the situation above. The reason is that the demand reduction caused by the inflation of price, or the demand increase caused by the price declining, will be multiplied for exponential function with the multiplicative reference effect. Therefore, enterprises will promote the commodity demand greatly by bring the price down to increase revenue.

\section{Comparison}

The four figures show significant changes in the optimal price paths. The characteristics are as follows. Firstly, the stronger the reference effect is, the more it influences the optimal prices and the total revenue. Secondly, the stronger the reference price is, the greater the total revenue and the optimal prices are. Thirdly, considering high or low demand with weak reference effect, the optimal price is lower than those in the benchmark model and the optimal revenue decreases when the reference price is low. The optimal price is higher than the benchmark price and the optimal revenue increases when the reference price is high. Moreover, when the reference price is in the benchmark interval, the optimal price and revenue remain unchanged. Considering the case of low demand with strong reference effect, we find that the optimal price is always lower than the benchmark price no matter how much the reference prices and the benchmark prices are.

How the firms in different markets choose the pricing strategies to maximize their revenue? The pricing strategies consist of markup or markdown policy as well as priceoriented or demand-oriented policy. For high demand, one should adopt the markup strategy to control demand and raise the total revenue. The higher reference price is, the faster the price rises. However, for low demand, one should promote demand by adopting the markdown strategy. When the reference price is higher, the price volatility is more obvious. The choice of the price-oriented policy or the demand-oriented policy plays an important role in the case of low demand. When the reference effect is strong, the demand amplification effect for price reduction is remarkable. Therefore, enterprises should focus on raising the demand level and make the lowprice strategy . Nevertheless, the first point for enterprises is to figure out how to utilize reasonable price for high revenue, i.e. enterprises should adopt price-oriented strategies in the case of weak reference effect.

\section{CONCLUSIONS}

The paper considers a dynamic pricing control model with the static reference price. The main results are as follows: the stronger the reference effect is, the more it influences the optimal prices and total revenue. The optimal prices and revenue increase with the reference price. The markup policy is effective in high demand situations and the markdown policy is effective in the low demand situations. In the situations with low demand and strong reference effect, the enterprises should adopt demand-oriented strategies. In other situations, they should adopt price-oriented strategies. Further research will take dynamic reference prices into consideration.

\section{ACKNOWLEDGMENT}

This paper is substantially supported by research grants from the National Natural Science Foundation of China (Grant No. 71571102 ).

\section{REFERENCE}

[1] W.C. Chiang, C.H. Jason, and X.J. Xu, "An overview of research on revenue management: current issues and future research," Int. J. Revenue Management, vol. 1(1), pp. 97-128, 2007.

[2] G. Gallego, G.V. Ryzin, "Optimal dynamic pricing of inventories control,” Manage. Sci. Maryland: Academic, vol. 40(8), pp. 999-1020, 1994.

[3] G. Gallego, G.V. Ryzin, "A multiproduct dynamic pricing problem and its applications to network yield management,” Oper. Res. Maryland: Academic, vol. 45, pp. 24-41, 1997.

[4] G. Gallego, M. Hu, "Dynamic pricing of perishable assets under competition,” Manage. Sci. Maryland: Academic, vol. 60(5), pp. 12411259, 2014

[5] A.J. Mersereau, D. Zhang, "Markdown pricing with unknown fraction of strategic customers,” Manuf. Serv. Oper. Manag. Maryland: Academic, vol. 14(3), pp 355-370. 2012.

[6] Y. Levin, J. McGill, and M.L. Nediak, "Optimal Dynamic Pricing of Perishable Items by a Monopolist Facing Strategic Consumers,” Prod. Oper. Manag. Oxford, vol. 19(1), pp. 40-60, 2010.

[7] M. Chen, Z.L. Chen, "Recent Developments in Dynamic Pricing Research: Multiple Products, Competition, and Limited Demand Information,” Prod. Oper. Manag. Oxford, in press.

[8] J. Popescu, Y. Wu, "Dynamic Pricing Strategies with Reference Effect," Oper. Res. Maryland: Academic, vol. 55(3), pp. 413-429, 2007. 